\title{
LA MODERNIZACIÓN AGRÍCOLA EN MÉXICO Y SUS REPERCUSIONES EN ESPACIOS RURALES
}

\section{Agricultural modernization in Mexico and its impact on rural spaces}

\author{
JESÚS GIL MÉNDEZ* \\ JOSEFINA VIVAR ARENAS**
}

Fecha de recepción: 25 de agosto de 2014 - Fecha de aprobación: 28 de octubre de 2014

\section{Resumen}

En este artículo se discuten aspectos relativos a los modelos de producción agrícola que se han puesto en práctica en México. Se hace un recorrido por las políticas agrarias creadas desde los años cuarenta, poniendo énfasis en los costos sociales y ecológicos que han tenido, particularmente desde los ochenta. Se discuten temas que se vinculan con la llamada agricultura moderna y tradicional, aspectos relativos a la relación sociedad-naturaleza, las críticas hacia el modelo de desarrollo rural moderno y las diversas alternativas que han creado grupos y comunidades para contrarrestar los efectos negativos de las políticas hacia el campo y la modernización agrícola.

Palabras clave: modo de producción agrícola, revolución verde, agricultura tradicional, agricultura moderna, relación sociedad-naturaleza.

\section{Abstract}

In this article are discussed aspects of agricultural production models that have been implemented in Mexico. A tour of agricultural policies created from the forties ago, emphasizing the social and ecological costs they have had, particularly since the eighties. Topics that are related to the so-called modern agriculture and traditional are discussed, aspects of society-nature relationship, criticism of the model of modern rural development and the various alternatives that have created groups and communities to counteract the negative effects of policy towards the countryside and agricultural modernization.

Keywords: mode of agricultural production, green revolution, traditional agriculture, modern agriculture, society-nature relationship.

* Doctor en Ciencias Sociales, El Colegio de Michoacán, A.C Profesor-investigador Universidad de la Ciénega del Estado de Michoacán de Ocampo, México. Correo electrónico: jegilme@gmail.com

** Doctora en Ciencias Sociales, El Colegio de Michoacán, A.C. Investigadora independiente. Correo electrónico: josefinavivar@gmail.com 


\section{Introducción}

El presente trabajo aborda algunas cuestiones referentes a los estilos o modelos de producción agrícola ${ }^{1}$ que se han efectuado en México, en particular en aquellas regiones donde desde mediados de la década de los cuarenta se aplicaron los supuestos de la denominada Revolución verde ${ }^{2}$. Se abordan los costos sociales y ecológicos que los estilos de desarrollo agrícola impuestos han provocado en las comunidades donde la actividad económica principal es la agricultura, también se discuten las estrategias que han adoptado los productores y campesinos ante el fracaso de las políticas agropecuarias que se pusieron en práctica sobre todo desde inicios de la década de los ochenta.

Entre las estrategias que analizamos y que consideramos una de las más importantes por sus diversos impactos en la vida de los agricultores, ha sido la migración a Estados Unidos. No obstante, las comunidades rurales recurren a diversas estrategias que les permiten hacer frente a sus necesidades. Al respecto, los investigadores argumentan que la vida rural en México se está reorganizando, y que productores y campesinos han desarrollado nuevas maneras de enfrentar sus desafíos a partir de la diversificación de sus actividades productivas (pluriactividad) y diversas formas de migración intermitente a las ciudades. A este fenómeno se le conoce ahora como Nueva Ruralidad (Gramont, 2009; Riaño \& Keilbach, 2009; Rubio, 1997; Torres, 2006).

Por lo anterior, tratamos de dar respuesta al siguiente cuestionamiento: ¿cuáles son las respuestas de los productores y campesinos frente a los modelos de producción agrícola que conformaron las políticas de desarrollo establecidas a partir de la revolución verde en México? Nuestro objetivo es analizar las relaciones que existen entre población y medio ambiente ${ }^{3}$ y sus implicaciones, comparando diversas formas de producir, en especial la agricultura tradicional con la moderna 4 . Puntualizamos las diferencias que existen en el modo agrícola tradicional y el moderno, tomando en cuenta la relación hombre-naturaleza que se da en estos dos modos de producir. Por último, hacemos un repaso a las múltiples respuestas y críticas que ha tenido el estilo de desarrollo rural moderno (modernización rural) ${ }^{5}$, y las diversas alternativas que se proponen desde diversos contextos.

\section{Contexto histórico}

Para Toledo (1999), existen tres modos históricamente determinados de apropiación de la naturaleza. El modo extractivo, propio de las primeras sociedades de pescadores, cazadores, recolectores; el modo campesino o agrario que llega con el inicio de la agricultura y la domesticación de plantas y animales, y el agroindustrial o moderno, producto de la revolución industrial y científica a partir del siglo XVIII. El modo campesino o agrario y el modo agroindustrial conforman las dos maneras fundamentales de usar los recursos en la actualidad. Esto significa que existen dos racionalidades productivas y ecológicas distintas, cuyas características principales se describen a lo largo de este trabajo.

Según Toledo, el cambio en las fuentes de energía dio lugar a la transformación del modo campesino al modo agroindustrial. Este cambio en el agro iniciado desde el siglo XVIII y continuado hasta el presente siglo XXI, es lo que se conoce cómo modernización rural (1999:72). Con el uso de nuevas fuentes de energía para extraer un mayor flujo de bienes de la naturaleza se modificó “(...) la articulación de los productores con los fenómenos y elementos de la naturaleza" (Ídem: 72). 
Cabe decir, que no obstante el predominio de estas dos maneras de usar los recursos en la actualidad, también existen entrecruzamientos en estas formas de apropiarse la naturaleza. Puede haber formas de producción que tengan características tanto del modo moderno como del modo tradicional de producción. Por lo tanto, en los paisajes rurales pueden coexistir diferentes grados de "campesinidad" o "agroindustrialidad", lo cual puede constatarse en la diversidad de productores que podemos encontrar en una región o una comunidad determinada.

Así, la agricultura moderna se ha sometido a un cambio acelerado debido al aumento de población y a las crecientes necesidades que ello implica, lo que ha traído consigo la introducción de nuevas tecnologías para intensificar la producción. El uso de tecnologías y de insumos altamente nocivos provoca el deterioro y agotamiento de los recursos. Con esta agricultura sólo se reconocen los beneficios monetarios a corto plazo y no sus implicaciones a largo plazo. Los efectos negativos de las políticas económicas pasadas y presentes han tenido que ver con el uso excesivo de insumos y la sobreexplotación agrícola que, además de modificar los sistemas de explotación agrícola, ha favorecido el monocultivo y los cultivos anuales en lugar de cambiar la rotación de cultivos y la variedad de los mismos. Otro efecto son las políticas de bajos precios a la producción agrícola, escasa inversión y altos costos de los insumos, además de la pérdida de la diversidad biológica que la producción agrícola moderna ha ocasionado en el ambiente natural (Escalante, 1993:60-63).

A diferencia del modo o estilo moderno de producción, en el tradicional se busca un equilibrio con el ecosistema y no una alta maximización de la producción con altos rendimientos. Para esta agricultura el suelo es un organismo vivo (Caba- nillas, 1992:6). Esta agricultura se caracteriza principalmente por el uso de diversas técnicas de producción que intentan mantener un equilibrio y una armonía con la naturaleza, tal armonía se encuentra empleando diversas prácticas, como pueden ser las siguientes: uso de semillas criollas, tracción humana o animal, cultivos intercalados, combinación de cultivos, se aprovecha el conocimiento empírico adquirido durante cientos de años por los cultivadores, principalmente indígenas. Este tipo de agricultura contrasta con la convencional en el sentido de que los campesinos gestionan los agroecosistemas con una orientación ecológica más correcta (Lampkin, 1988:15).

\section{Los inicios de la Revolución verde}

La situación del agro mexicano da un vuelco con la revolución ocurrida a inicios del siglo pasado, y las haciendas, que habían sido la principal organización en torno a los procesos agrícolas y productivos terminan por desmoronarse. Así, se crean las condiciones para que al final del movimiento armado se inicie el reparto de las haciendas; no obstante es en el periodo presidencial de Lázaro Cárdenas, con el establecimiento de los ejidos, que se impulsó masivamente el reparto agrario, lo que significó que las tierras en manos de las haciendas quedaran en los ejidos. Al final del reparto agrario, sobre todo en la década siguiente, se dará inicio a un proceso de modernización agrícola en diversas regiones del país que se conoce como revolución verde, del cual nos ocuparemos enseguida.

El proceso de transformación del modo campesino al modo agroindustrial con todas sus consecuencias ecológicas, sociales y culturales, se empieza a dar a gran escala en México desde mediados de la década de los cuarenta con la llamada revolución verde ${ }^{6}$. Esta se basó sobre 
todo en una producción constante ${ }^{7}$, sostenida en un modelo de agricultura y ganadería intensivo en donde prevalece la utilización de materiales y energía que proviene de fuentes fósiles de existencia cada vez más limitada. Algunos de estos materiales son los fertilizantes y pesticidas, así como los combustibles utilizados para el uso de máquinas. Otras características de la revolución verde son la especialización, al impulsarse el monocultivo con la selección de variedades de alto rendimiento utilizando semillas mejoradas; el empleo de grandes dosis de fertilizantes y la intensificación de las labores agrícolas y la utilización de riego, todo ello con el fin de incrementar la producción.

La revolución verde en la agricultura requirió la expansión del crédito y el comercio. Para adquirir el paquete tecnológico, campesinos y productores tuvieron que convertirse en sujetos de crédito. La alta producción hizo crecer la intermediación comercial. Para que el éxito de la revolución verde fuera redondo hubo que construir grandes sistemas de riego, sobre todo en el noroeste, noreste y partes del centro del país, lugares donde se crearon verdaderos polos del desarrollo agrícola capitalista en México.

En las regiones donde los supuestos de la revolución verde se establecieron con más intensidad, también se ha dado lugar a un mayor deterioro ambiental, eso se puede observar, por ejemplo, en la reducción de la fertilidad de las tierras que son sometidas a una producción intensiva. Esta sobreexplotación de recursos trae también como consecuencia la contaminación de mantos freáticos, enfermedades e intoxicaciones por el consumo de productos contaminados y vivir en continua exposición tanto en el trabajo, como en los lugares de residencia contiguos a espacios que son infestados de diversos productos nocivos para la salud.
La justificación de este modelo es satisfacer la demanda urbana e industrial de los productos agropecuarios, requiriendo para ello el aumento de los volúmenes de productos en el menor tiempo posible. Con este modelo los ecosistemas naturales son destruidos y convertidos en sistemas artificiales especializados, que generan en forma masiva productos competitivos en el mercado para alcanzar una alta rentabilidad. Para que haya una "modernización" agrícola de este estilo no solo se debe introducir un nuevo paquete tecnológico, también tiene que haber una gran infraestructura hidráulica y de carreteras que la soporten, además de mecanismos de control social del campesinado que aseguren una estabilidad política.

De acuerdo con estos argumentos, se impulsó la transformación de la agricultura tradicional en un sector económico "moderno"8. Pero como veremos más adelante, este modelo de agricultura intensiva es cada vez más costoso ambientalmente; la contaminación, la degradación de los suelos, la deforestación, la reducción de la diversidad biótica y el agotamiento progresivo de los recursos naturales son algunos de los retos que tienen que enfrentar las comunidades locales y la sociedad en general.

\section{El milagro mexicano y el neoliberalismo}

Desde mediados de la década de los cuarenta hasta mediados de los setenta, se logró lo que se llamó el milagro mexicano, que se caracterizó por la elevación del nivel de vida material, tanto por los servicios sociales a los que se tuvo acceso, como por el mejoramiento del poder adquisitivo de amplios segmentos de la población. Sin embargo, esto se truncó desde finales de los setenta con la puesta en marcha del neoliberalismo ${ }^{9}$, provocando la polarización en 
la sociedad mexicana y los daños que esto ha traído al medio ambiente ${ }^{10}$ (Barkin, 1997:235).

Aun con los beneficios que pudo haber tenido el milagro mexicano para algunos sectores de la población ${ }^{11}$, con el proyecto implementado en México desde los cuarenta, el sector rural quedó rezagado y sólo algunas áreas dedicadas a la agricultura comercial fueron protegidas con subsidios, infraestructura para la producción y comercialización, crédito, asistencia técnica, etc. No obstante, los sectores agrícolas menos favorecidos (ejidatarios, pequeños productores, minifundistas, campesinos), sostuvieron el abasto de materias primas para el país y con ello impulsaron el desarrollo industrial. Esto generó una agricultura polarizada y una acelerada expansión urbana. Se consolidó un pequeño grupo capitalizado de productores que acaparó las mejores tierras, concentrando los medios de producción y aplicando las tecnologías modernas para producir bienes de exportación. En el otro extremo quedó la mayoría de los campesinos sin apoyos, en las tierras de temporal, produciendo granos básicos y sin infraestructura (Carabias, 1990).

Hacia los años ochenta, se inició en México un proceso de reorientación de las políticas de desarrollo nacional que fomentaron el establecimiento del modelo neoliberal. Para Olvera, "la transformación neoliberal en el campo fue muy radical e implicó no sólo el fin de la omnipresencia estatal, sino también la necesidad de una reforma constitucional para abrirle las puertas a un mercado legal de tierras ejidales y atraer la inversión privada" (1997:76). Esta transformación en el campo implicó, entre otras cosas, que los precios de los productos agrícolas cayeran, se canceló la política de precios oficiales para la mayor parte de los productores y la producción capitalista moderna perdió los antiguos beneficios del Estado. La inserción internacional de la agricultura mexicana y los efectos de los acuerdos de libre comercio tuvieron como consecuencia la reducción de la población en el agro, la pauperización de los campesinos y su exclusión del proyecto de desarrollo nacional; se produjeron profundos cambios demográficos en el sector rural, así como en la composición de los grupos domésticos, cuyos integrantes se vieron forzados a migrar para obtener empleos en otro oficios no agrícolas. Diversos investigadores han denominado a este proceso de acelerada transformación del contexto rural, como nueva ruralidad. De acuerdo con este enfoque:
"(...) los nuevos escenarios en el campo se caracterizan por una menor diferenciación entre lo rural y lo urbano en términos de productos y servicios, tanto en mercados laborales como en patrones residenciales, cambios en mercados de trabajo rurales marcados hacia un menor peso de las actividades agropecuarias, flexibilización laboral, y por ende, cambios en estrategias de ingreso de grupos domésticos, con una acentuación a la pluriactividad como común denominador" (Riaño \& Keilbach, 2009:81).

Además, como parte de los efectos demográficos que estas trasformaciones han causado, en el campo mexicano las actividades agrícolas son desempeñadas generalmente por adultos mayores (Gil, 2012) y cada vez más mujeres (Vivar, 2012). Frente al contexto de crisis económica, de colapso de instituciones y de mecanismos que habían estructurado la vida rural, los campesinos crearon salidas propias, las estrategias de sobrevivencia se trasladaron al núcleo familiar, cuyos integrantes recurrieron a la migración definitiva o temporal y a la pluriactividad. Esto causó diversos efectos socioculturales en los planos locales y en la organización campesina (Vivar, 2012). Por ejemplo, las zonas de agricultura comercial en 
zonas mestizas sufrieron las consecuencias de la crisis, lo que las orilló a crear estrategias adaptativas, una de las cuales ha sido principalmente la emigración a Estados Unidos (Gil, 2012). De acuerdo con Olvera, estas formas de adaptación dependen de las pautas previas de integración de los campesinos al mundo urbano (1997:85).

Calva (1989), señala que lo sucedido en el campo mexicano desde 1983, con la implementación del modelo neoliberal, ha sido un proceso de regresión económica y técnica; los precios adversos, falta de créditos, retiro de subsidios, entre otras cosas, han hecho que México dependa del exterior. Desde la década de los ochenta, la falta de desarrollo en el campo provocó el deterioro del sector. Algunas de sus manifestaciones sociales fueron el desempleo y la migración a los centros urbanos y a Estados Unidos. En suma, las políticas macroeconómicas que han llevado a cabo los gobernantes desde esos años, han dañado considerablemente a los productores y campesinos mexicanos. Estas políticas han promovido las exportaciones y un adelgazamiento del Estado, lo que ha provocado menos apoyo al sector agrario, caída de los salarios reales y la contaminación ambiental.

Con tales políticas aplicadas, tanto la agricultura de temporal como de riego han sido severamente golpeadas, y aunque para algunos estudiosos (Barkin, 1997), la agricultura en los distritos de riego ha contado con el apoyo financiero para cultivar frutas y verduras e integrarse a los mercados internacionales, esto no ha sido homogéneo para todos los distritos. Se debe considerar que en cada región existen particularidades muy específicas, y las maneras en que los recursos son apropiados, distribuidos y aprovechados, son variadas. En algunos de los distritos o módulos de riego, los productores han aprovechado sus condiciones tecnológicas, ambientales y los apoyos que han recibido del Estado para convertirse en productores capitalistas, enriquecidos gracias a la producción intensiva de cultivos de exportación. Pero existen también módulos de riego donde los productores poseen parcelas cuya extensión de terreno suele ser muy pequeña ${ }^{12}$ (desde media hasta cinco hectáreas) y, aunque cultiven hortalizas y productos de exportación, quienes se suelen beneficiar de este modo de explotar la tierra son los empresarios dedicados a la agroindustria y los intermediarios, y no los productores (aunque esto puede variar según la región o comunidad).

Es así que los productores que más han aprovechado estas ventajas comparativas son aquellos agricultores que poseen y cultivan grandes extensiones de tierra. Esto se debe, sobre todo, a que se condiciona el desarrollo al imponer formas más extensivas de uso del suelo, sobreexplotándolo. De manera que, aunque muchos productores minifundistas cuenten con recursos y condiciones tecnológicas para producir cultivos de exportación, reciben muy poco apoyo, y los beneficios económicos que obtienen llegan a ser en muchos de los casos muy parcos o inclusive con pérdidas ${ }^{13}$.

\section{Las criticas al modelo agrícola desarrollado en México}

Aunque las sociedades a lo largo del tiempo siempre han hecho uso de los ecosistemas ${ }^{14}$ explotando los recursos naturales, en la actualidad su explotación es cada vez más intensiva y de mayor magnitud. La sociedad contemporánea se sustenta en una base que exige una cantidad cada vez mayor de recursos naturales, generalmente utilizados de manera ineficiente. 
Este ritmo acelerado en el uso de los recursos "(...) supera los tiempos normales de regeneración y asimilación del sistema natural, resultando en un agotamiento acelerado de recursos y degradación del medio" (Bifani, 1997:300).

La aplicación indiscriminada de cualquier tecnología puede causar daños irreversibles muy costosos ecológica y económicamente. Los efectos sobre el medio ambiente son producto de la artificialización de la agricultura, debido a que ésta descansa sobre recursos como agua y suelo, que también se deterioran y agotan. La agricultura actual, por un lado desperdicia el gran potencial que ofrece la diversidad ecológica y, por otro, fuerza a la naturaleza “(...) a producir en contra de sus propias leyes de funcionamiento" (Carabias, 1990:344), al homogeneizar la producción e ignorar la variabilidad ambiental. Se sigue produciendo con una base natural cada vez más deteriorada y con tecnologías que profundizan este daño. ¿Cuanto tiempo más se podrá sostener la producción con las actuales tecnologías que siguen degradando los recursos naturales? Para Carabias (1990), las políticas de producción agropecuaria instrumentadas hasta la fecha, han dejado de lado los problemas que estas actividades causan sobre la naturaleza. Según esta investigadora, la dimensión ambiental no ha sido incorporada en las políticas de desarrollo rural. Hace unas décadas esta cuestión no significaba un problema porque el daño no era evidente, además que se creía que los recursos naturales eran inagotables, y aunque ahora hay conciencia de los daños que han causado ciertas formas de producción, no se aplican los correctivos necesarios para encontrar solución frente al problema.

La crisis agrícola por la que atraviesa el país es muy grave, esto ha tenido que ver con la anulación de subsidios y apoyos a productores. La combinación de la crisis y el deterioro tienen severas consecuencias para la población campesina, el patrimonio biológico y la soberanía nacional (Torres, 1995). Con tal modelo se propició una ruptura en la visión de la naturaleza como ente vivo, la tierra se consideró como una máquina, separando artificialmente al proceso agrícola de sus conexiones con lo ecosistemas (Toledo, 1996). Se manipuló a la tierra de acuerdo con los desarrollos físicos y químicos logrados en laboratorios y trasladados después al campo. Esta visión del conocimiento científico parcelario ha sido fuertemente criticada, lo que ha impulsado la creación de nuevos paradigmas que contradicen los cimientos en los que se basó la explotación agrícola.

De acuerdo con González (1996), la atención en los efectos de los insecticidas en el medio ambiente, el carácter ineficiente de la agricultura desde el punto de vista del uso de la energía, los efectos no deseados de este modelo de agricultura manifestados en los impactos negativos sobre los ecosistemas, dio impulsó la agroecología ${ }^{15}$. De esta manera, las propuestas han ido desde la generación y promoción de nuevas disciplinas como la agroecología y otras alternativas, con un enfoque característico del desarrollo sustentable ${ }^{16}$, que se discutirán más adelante.

No se han tomado las medidas necesarias para evitar el deterioro, agotamiento o extinción de los ecosistemas. Los planes solo atienden el presente sin preocuparse del largo plazo. Los recursos se agotan porque se extrae de ellos una producción mayor de lo que la propia naturaleza es capaz de reproducir, bajo el argumento de que las actuales formas de producción son las únicas posibles de aplicarse. Algo que se debe reconocer es que los ecosistemas tienen 
un determinado ritmo y si la extracción supera este ritmo se atenta contra el capital natural, hasta llegar a su agotamiento (Carabias, 1990). Meadows (1972), coincide con esta idea cuando señala que existen muchos límites para la producción de alimentos, uno es la tierra cultivable y otro es la disponibilidad de agua. De manera que, aunque se decidiera pagar los costos necesarios para ganar nuevas tierras 0 incrementar la productividad de la tierra ya cultivada, el crecimiento de la población provocaría un punto crítico, que hace que sea cada vez más costoso superar los sucesivos puntos críticos que se irían presentando con cada duplicación de productividad. A esto le llama ley de costos crecientes. Esto significa que se tienen que utilizar cada vez más tractores, plaguicidas, fertilizantes, insumos y recursos para alcanzar nuevos aumentos en la producción, con el fin de satisfacer la demanda urbana e industrial de los productos agropecuarios, que requiere el aumento de los volúmenes de producto en el menor tiempo posible. Con este modelo, los ecosistemas naturales son destruidos y convertidos en sistemas artificiales especializados, que generan en forma masiva productos competitivos en el mercado.

\section{Consecuencias sociales del estilo de producir moderno}

Enseguida, discutimos los efectos sociales del modelo agrícola de desarrollo instrumentado en México a partir de los ochenta ${ }^{17}$, y la influencia que ha tenido en el incremento de la migración, particularmente en aquellas regiones de agricultura intensiva, que fueron de las más afectadas por la política neoliberal.

En este contexto, se debe precisar que el aprovechamiento de los sistemas agrarios o agroecosistemas ${ }^{18}$ no depende sólo de presiones ambientales, sino también de relaciones sociales que establece la explotación agrícola, pues ella no está determinada solamente por factores de origen biótico o ambiental. Factores sociales como cambios en los regímenes de tenencia de la tierra, el tamaño de la familia, pueden afectar a los sistemas agrícolas tanto como una plaga, una sequía, etc. En este sentido:

\section{“(...) la producción agraria es el resultado de las presio- nes socioeconómicas que realiza la sociedad sobre los ecosistemas (...) y este principio resulta fundamental puesto que permite integrar en un enfoque multidiscipli- nario las prácticas sociales desde la perspectiva de su impacto ambiental" (González, 1996:411).}

En México, la ejecución de los programas de ajuste estructural y reconversión económica junto con la reestructuración productiva a inicios de los ochenta, creó nuevas condiciones para la emigración, por lo que "en este contexto de inequidad y precariedad salarial, la migración a Estados Unidos se ha convertido en una alternativa laboral no sólo atractiva, sino real y posible para cada vez más sectores de la población de diversas regiones del país" (Canales, 2002:56). La falta de oportunidades económicas en el área rural fuerza la migración de miles de personas, en especial jóvenes, contribuyendo en muchos lugares a la feminización y ancianización del agro (Altieri, 2002:283). Sobre esto, cabe señalar que la emigración de campesinos y productores a Estados Unidos puede afectar la manera de explotar los recursos agrícolas. Tal como lo señala Hogan, la migración en cuanto significa desplazamientos de un lugar a otro, puede cambiar radicalmente la relación entre población nativa o residente y su medio ambiente" (Hogan, 2002:168). Esta relación entre la migración y el medio ambiente: 
“(...) implica tratar la distribución de los recursos naturales en el espacio; el uso histórico de estos recursos por parte de las poblaciones humanas y su agotamiento o degradación. También significa tratar las consecuencias de cambios ambientales provocados por la actividad humana debido a la movilidad" (Ídem: 167).

Esto significa que tanto la inmigración como la emigración pueden traer diversas consecuencias en la manera de aprovechar los recursos agrícolas. La magnitud de tales consecuencias dependerá de la presión y el modo de uso de los recursos, y el factor demográfico puede ser un agente importante en ello. Por ejemplo, puede haber casos en que la emigración de campesinos y productores a Estados Unidos reduzca la presión sobre los recursos y se mantengan las propiedades originalmente dotadas, lo que impide su parcelamiento y la multiplicación de minifundios (en algunos casos, esto reduce la posibilidad de ampliar y diversificar las actividades productivas). Ello, permitiría al agricultor no dividir sus recursos y mantener sus actividades productivas en una determinada superficie. Lo contrario se puede ver en regiones con poca migración, donde la presión sobre los recursos provoca la reducción de la superficie agrícola del campesino y, por lo tanto, lo que produce, al mismo tiempo que le impide diversificar su producción en una superficie de terreno cada vez más pequeña ${ }^{19}$.

Podemos asegurar que la profunda crisis que vive el campo mexicano generada por la falta de capacitación, de apoyos, la corrupción de sus instituciones, ha causado la migración de miles de productores quienes en muchos casos ya no tienen interés por la tierra, lo que provoca muchas veces que el conocimiento tradicional no sea transmitido en la actualidad hacia los jóvenes como era en el pasado. Estos rechazan los valores tradicionales como símbolo de atraso frente a la supuesta modernidad, y desestructuran en muchos casos, la organización tradicional. Si bien es cierto que el fenómeno migratorio puede causar una ruptura entre los campesinos viejos y los jóvenes, lo que podría significar la pérdida de la continuidad de viejas formas de identidad y de la renovación de liderazgos, también se crean condiciones para que surjan nuevos actores locales, con sus propios intereses particulares, y donde se puede originar un mayor apego a la cultura comunal y un mayor reforzamiento en los lazos comunales y en la creación de nuevos intermediarios culturales.

Por último, en la actualidad cada vez es más difícil que los agricultores cubran sus necesidades de ingreso a través de la actividad productiva, esto lo compensan con lo que obtienen de otras actividades laborales. Es así, que los productores multiplican sus estrategias para poder subsistir ante los embates de la globalización y el neoliberalismo. Concluiremos estas ideas mencionando que, las formas de apropiación de los recursos pueden contribuir a incrementar o disminuir el deterioro ambiental, y esto tiene una relación directa con la distribución de los recursos en la población. Es importante señalar que la manera de utilizar los recursos agrícolas puede impactar de la misma forma tanto en pequeñas como en grandes propiedades.

\section{Consecuencias ecológicas del estilo de producir moderno}

Como ya se mencionaba, el funcionamiento de la sociedad moderna se sustenta en la utilización masiva de los elementos naturales existentes en forma directa o a través de complejos procesos de transformación. Tal enfoque ha orientado históricamente la utilización de los 
recursos naturales de una manera reduccionista. Esto se ha puesto de manifiesto en dos hechos precisos: "la consideración del producto per se, desligado de su relación con el sistema del cual es parte, y la forma en que se han valorado los recursos" (Bifani, 1997:302). El tipo de desarrollo agrícola impuesto no sólo ha traído consecuencias en el renglón social, también ha dejado su huella en el ambiente deteriorando muchas veces los recursos naturales, en ocasiones de manera irreversible. Las políticas agrícolas instrumentadas y los programas aplicados no han tomado las medidas necesarias ni aplicado las tecnologías idóneas para evitar el deterioro ambiental (Carabias, 1990).

Tenemos entonces que el estilo de producción rural moderno ha traído diversas transformaciones y modificaciones en los ecosistemas naturales y sus servicios ambientales. Esto se ha debido, básicamente, a un desarrollo rural no planificado que ha utilizado extensivamente los recursos naturales con la concepción de que son ilimitadamente renovables (Carabias, 2002:258). La falta de planeación con una visión no sustentable, junto con diversos procesos sociales, económicos y ambientales ha generado un sinnúmero de impactos severos en la naturaleza, que se han intensificado a partir de la segunda mitad del siglo (lbíd.). Para Altieri, en la década de los ochenta "(...) el deterioro de la salud y la degradación ambiental fueron problemas que continuaron aquejando a la población rural" (Altieri, 2002:281). Se hizo más evidente "(...) que los modelos convencionales de modernización de la agricultura, basados en monocultivos dependientes de un alto nivel de insumos agroquímicos, era un modelo no viable desde el punto de vista social y ecológico" (Ibíd.). De esta manera, la agricultura comercial y de exportación ha llevado al incremento en el uso de productos agroquímicos, muchos de ellos prohibidos en países desarrollados por razones ambientales o de salud humana, pero consumidos todavía en los países subdesarrollados.

Uno de los muchos problemas que enfrenta la agricultura de exportación es la producción de hortalizas y otros cultivos utilizando aguas negras sin tratar, lo que ha traído serias consecuencias para la población, tanto para quienes trabajan en estos campos agrícolas como para quienes consumen estos productos (Barkin, 1997:237). Así, para González,

\footnotetext{
"(...) nuestro modelo de consumo y la agricultura intensiva-capitalista que lo hace posible, cuyo principal objetivo no es alimentar a los seres humanos sino producir el máximo beneficio posible, resulta moral, social y ecológicamente insostenible. Los pobres mueren de hambre o desnutrición para que nosotros podamos comer en exceso alimentos que perjudican nuestra salud; mientras la tierra se convierte en desierto y los recursos se agotan" (1996:402).
}

La utilización de los recursos enfrenta una doble problemática: su agotamiento y deterioro se deben examinar desde la perspectiva de la existencia de recursos naturales conocidos y las leyes naturales que regulan su proceso de producción, así como de la capacidad del medio “(...) para regenerarse y absorber el impacto de la actividad humana (...) y también, por la forma en que el sistema social lleva a cabo sus actividades, traduciéndose en formas específicas de gestión ambiental" (Bifani, 1997:300). De este modo, la disponibilidad de recursos depende de un estilo de vida y un patrón de desarrollo. Es cierto que el modelo agrícola moderno aumentó la productividad en muchos cultivos, y que hubo una gran producción de alimentos, pero la insuficiencia de alimentos no se da por falta de producción sino por la distribución desigual de los recursos, y no es posible que se solucione esta 
falta de producción con solo medidas técnicas. La imposición de este modelo provoca mayores diferencias sociales entre productores, además del deterioro del ambiente, que se traduce en el agotamiento de los recursos, la contaminación del medio y los daños directos al trabajador, pues los agricultores tienen acceso a los recursos más depredadores de la naturaleza.

\section{La relación sociedad-naturaleza y el enfoque ambiental}

Hemos analizado los diversos impactos sociales y ecológicos que han tenido los modelos de desarrollo agrícolas impuestos en México desde el siglo pasado. En contraste con estos modelos han existido (y existen todavía) desde tiempos remotos, diversas formas de aprovechar los recursos que no tienen nada que ver con las maneras de apropiarse los ecosistemas que se imponen desde fuera. En México, diversos grupos indígenas y campesinos han vivido y convivido con la naturaleza desde ya varios siglos en una relación armoniosa, lejos de las formas capitalistas y consumistas de aprovechar los recursos hoy día. La percepción de la naturaleza que tienen estos grupos se relaciona con su sobrevivencia, de ahí que las prácticas productivas deben comprenderse desde el punto de vista de las diferentes formas de concebir el mundo.

Eckart (1996), señala que en los grupos de campesinos (que practican formas de producir diferentes a la agricultura moderna) no existen límites claros entre naturaleza y sociedad, el humano es parte de ella y la naturaleza parte de él. La naturaleza es un espacio sagrado que tiene una lógica oculta que no puede ser controlada por los humanos, por lo que se debe establecer un intercambio simbólico para recibir los alimentos. Se interviene en el territorio, pero como si fuera prestado. Así, nos dice Eckart, en los rituales se dan regalos para tratar de restablecer un equilibrio, se pide perdón, se explica el porqué del desmonte y del establecimiento de un cultivo. Si no se pide en esta relación de reciprocidad, se corre el riesgo de perder la cosecha, de enfermar y hasta de morir. Esto nos muestra que no hay una escisión entre sociedad y naturaleza, sino que la relación existente del ser humano con la naturaleza y las transformaciones que se derivan de ello, son parte de los fenómenos sociales.

Esta relación sociedad-naturaleza ha logrado que diversos grupos indígenas, campesinos y agricultores sobrevivan ante las crisis agrícolas. Por ejemplo, Toledo (1993) menciona que existen diversas estrategias indígenas de manejo de los ecosistemas, en donde el resultado es un sistema integrado de varias unidades productivas, en el que se combinan elementos de origen prehispánico con otros derivados del contacto europeo y moderno. Resalta aquí el papel de las comunidades indígenas en el mantenimiento y manejo de las áreas naturales, así como la diversidad lingüística en estrecha relación con la diversidad biológica. Podemos deducir de estos planteamientos que, aun a pesar del desequilibrio ecológico que existe actualmente, hay prácticas agronómicas que rescatan principios en sistemas que mantienen viva la tierra, estos métodos recuperan técnicas tradicionales de los agricultores, actualizándolas y mejorándolas con algunas técnicas modernas que son respetuosas del ambiente. El principio fundamental de la agricultura tradicional es que busca un equilibrio con el ecosistema y no una alta maximización de la producción con altos rendimientos. Tal agricultura se caracteriza por el uso de diversas técnicas de producción que 
intentan mantener un equilibrio y una armonía con la naturaleza.

Se ha propuesto entender la relación sociedad-naturaleza en el marco de un proceso evolutivo en donde ambas interaccionan en el tiempo, sin poderlas desligar y entender una sin la otra. Para entender esta relación mutua en todos los niveles, Toledo \& González (1994), proponen el concepto de metabolismo social ${ }^{20}$ como parte central de este nuevo enfoque. Para los autores, en cualquier discurso renovado se deben replantear los objetivos que hasta ahora los científicos sociales han presidido. El enfoque ambiental aporta a las ciencias sociales la preocupación por la sustentabilidad, por la condición material de toda relación social. Los autores señalan que estos nuevos enfoques no pretenden explicarlo todo desde una óptica ambiental, debido a que las relaciones sociales son complejas y no se pueden reducir a análisis físico-biológicos.

\section{Perspectivas y propuestas}

¿Qué alternativas o propuestas pueden plantearse ante este panorama desolador de utilizar y desperdiciar los recursos? Enseguida enumeramos algunos planteamientos de varios estudiosos que nos parecen que pueden llevarse a cabo, y que de constituirse podrían (y de hecho lo están haciendo) generar cambios que disminuyan los efectos adversos de nuestro estilo de producción actual, reduciendo los costos sociales y ecológicos que tal estilo conlleva.

En contraposición con la tendencia dominante que tiende a la especialización del conocimiento y como respuesta de los limitados análisis del enfoque analítico-parcelario, un nuevo enfoque surge desde mediados del siglo $\mathrm{XX}$ que intenta integrar las ciencias sociales y humanas con las naturales (Toledo, 1999:55-56). Esto ha dado lugar al surgimiento de las disciplinas híbridas ${ }^{21}$ que buscan ofrecer información para remontar la crisis ecológica. En las disciplinas híbridas se adopta un enfoque de “(...) integración del estudio sintético de la naturaleza (la ecología) con diferentes enfoques dedicados a estudiar el universo social o humano" (Ídem: 58). De esta manera, se abren múltiples perspectivas que responden a los intentos por encontrar soluciones a la crisis ambiental. Para Leff (1997), la crisis ambiental es sobre todo un problema de conocimiento, lo que lleva a repensar al mundo de una manera compleja. Así, la crisis ambiental es una crisis de la civilización, y su solución no se puede encontrar "(...) por la vía de la racionalidad teórica e instrumental que construye y destruye el mundo" (Leff, 1997:8), sino que implica un proceso de desconstrucción y reconstrucción del pensamiento. Con el pensamiento complejo se cuestiona el entendimiento con que la civilización occidental ha comprendido el ser, "(...) la ciencia y la razón tecnológica con las que ha sido dominada la naturaleza y economizado el mundo moderno" (Ídem: 11). En síntesis, se interroga el conocimiento del mundo que ha buscado la uniformidad y la homogeneidad.

Toledo (1996), propone elaborar un uso ecológicamente correcto de los recursos que sea económicamente rentable y justo, que la planeación y el manejo ecológico se realicen mediante el diálogo y la investigación participativa. La conjunción de los conocimientos de los investigadores herederos de la tradición científica europea y los saberes y prácticas acumulados por los habitantes de las culturas indígenas y campesinas locales. Integrar planes de manejo comunitarios y microrregionales que 
sean resultado de la integración de métodos y tecnologías avanzadas, con las experiencias acumuladas de los productores locales. Mejorar la estrategia de las culturas locales y no destruir su experiencia y sustituirla por formas modernas de expoliación y depredación de los recursos naturales. La estrategia que propone Toledo se basa en el uso múltiple de los recursos naturales, producir con base en los principios de la diversidad de recursos que sobre la especialización, al utilizar la mayor cantidad de productos que ofrece un ecosistema a lo largo del año. Para Toledo, Occidente todavía impide la integración de saberes que pueden ayudar a salir de la crisis actual. Esto implica un rompimiento epistemológico, cultural, social y civilizatorio, y la creación de lo que llama ciencia posnormal.

Así mismo, una alternativa que ofrece Barkin es lo que el llama economía de guerra, donde plantea la reconstrucción del mercado interno, proponiendo la autosuficiencia alimentaria, creando trabajo, movilizando recursos locales, rescatando los recursos naturales, el equilibro ambiental y la capacidad productiva. En síntesis, que los productores fortalezcan su capacidad de autoabastecimiento, sustituyendo importaciones cuando se pueda y creando nuevas industrias (1997:237).

Es necesario cambiar la racionalidad en la forma de cultivar la tierra, ya que no sólo se trata de mejorar la productividad del suelo, sino de recuperar lo que se ha destruido. Los ecosistemas deben trabajarse mediante una agricultura ecológica que revierta el deterioro y proteja los recursos naturales y a la sociedad. En una nueva relación sociedad-naturaleza, la conservación de la naturaleza implica la preservación de la sociedad con un sentido de armonía y respeto con nosotros mismos y la naturaleza.
Es necesario entonces un desarrollo sustentable que logre el equilibrio entre hombre y naturaleza, que ponga fin al uso irracional de los recursos naturales y que haya una mayor participación de todos los individuos como gestores y promotores de su desarrollo humano.

\section{Conclusiones}

El estilo de desarrollo actual, caracterizado en lo político, económico y tecnológico como un estilo centralizador, alienta las grandes concentraciones urbanas con una gran demanda y un consumo mercantilista, además de la migración de campesinos a centros urbanos y a otros países, provocando con ello en múltiples niveles, el desarraigo y la pérdida del conocimiento tradicional y de diversas formas culturales e identitarias de los pueblos campesinos. Este estilo de desarrollo ha creado demandas irracionales de energía, lo que hace necesario que se requieran nuevas tecnologías que sean más respetuosas del ambiente, menos consumidoras de energía. Se deben plantear entonces, diferentes condiciones para el sector rural y para el sector urbano industrial.

Los estilos de desarrollo impuestos hasta ahora han negado las diferencias culturales, climáticas y geopolíticas de los países, y las diferencias regionales existentes dentro de cada país. No se ha tomado en cuenta que las sociedades se conducen por caminos diferentes en su estilo de desarrollo. Por otro lado, no se debe pensar de manera nostálgica y creer que sólo los sistemas tradicionales de cultivo son los más viables, ni tampoco se debe satanizar a la agricultura convencional. Se deben de considerar los logros que ha tenido este tipo de agricultura y rescatar aquellas prácticas que sean ecológicamente viables, combinando en algunos casos 
tecnología tradicional y moderna. No se trata de reivindicar todas las tecnologías tradicionales, pero sí extraer los principios de las que resulten más eficientes y también aprender de las que resulten con fallas. Tal cómo señala Altieri,

\section{“(...) necesitamos modelos de agricultura sustentable que combinen elementos de ambos conocimientos, el tradicional y el moderno científico. Complementando el uso de variedades convencionales e insumos comer- ciales, con tecnologías ecológicamente correctas se puede asegurar una producción agrícola más susten- table" (citado en González, 1996:436).}

Diversas alternativas rentables a nivel socioecológico comienzan a ser ejemplo para aquellos preocupados por el deterioro ambiental, muchos proyectos productivos han sido puestos en marcha y logrado éxito en diversas comunidades agrícolas y campesinas. Se debe ampliar este éxito a otras comunidades para que se vayan generando nuevas formas de producir y de relacionarse con la naturaleza, que impliquen un mayor respeto y que cada vez haya una relación más armoniosa entre la sociedad y la naturaleza. Esto implica “(...) una nueva comprensión del mundo que incorpora los conocimientos y saberes arraigados en cosmologías, mitologías, ideologías, teorías y saberes prácticos que están en los cimientos de la civilización moderna (...)" (Leff, 1997:14).

Lo que involucra cuestionar la ciencia y el conocimiento que ha prevalecido hasta ahora. La complejidad ambiental envuelve un cambio de mentalidad y pensamiento, una transformación del conocimiento para construir un nuevo saber y una nueva racionalidad donde se construya un mundo sustentable, de equidad (Ibíd.).

El Estado ha olvidado la agricultura. Si no se apoya el crecimiento interno muy pocas alternativas van a tener los millones de productores y campesinos que se debaten ahora entre la miseria y la pobreza. El Estado debe promover y consolidar las estructuras organizativas de los productores e implementar apoyos en la comercialización de sus productos, y también incentivar aquellas formas de producción que respetan al medio natural. Podemos ver entonces que diversos sistemas que dañan a la ecología y la sociedad están siendo objeto de crítica desde hace décadas, y que poco a poco una nueva forma de pensar se va abriendo brecha ante modelos de desarrollo injustamente impuestos. 


\section{Notas}

${ }^{1}$ Para el presente trabajo, se entenderá por modo o estilo de producción (o desarrollo) agrícola, las diferentes formas de explotar y aprovechar los recursos agrarios; lo que implica la manipulación o apropiación de los ecosistemas naturales para la producción de bienes, empleando para ello ciertos saberes, energía e instrumentos de producción.

${ }^{2}$ Se describirán en otro apartado las características fundamentales de la Revolución verde.

3 Para los fines de este trabajo, nos basaremos en la definición que hace Sunkel sobre medio ambiente: "el ambiente biofísico natural y sus sucesivas transformaciones artificiales así como su despliegue espacial" (Sunkel, 1980:13). En otras palabras, es la flora, fauna, la superficie disponible para la actividad humana; el medio ambiente artificializado y las interacciones que se dan entre diversos elementos como el aire, agua, tierra y la sociedad.

${ }^{4}$ Más adelante describimos las principales características de estos tipos de agricultura.

${ }^{5}$ La modernización o el desarrollo rural se concibe como la transformación productiva de las formas campesinas tradicionales o preindustriales en modalidades agroindustriales o modernas. De esta manera, la modernización rural corresponde al momento en que "(...) el modo campesino de apropiación se ve transformado o sustituido por el modo agroindustrial" (Toledo, 1999:73). Esta transformación es lo que Sunkel llama desarrollo moderno, el cuál se basa en "(...) la gradual especialización del trabajo y los correspondientes cambios tecnológicos, así como en un aumento en la utilización de energía no humana, lo que ha permitido aumentar su productividad y con ello generar un excedente por sobre lo necesario para reproducir la fuerza de trabajo" (Sunkel, 1980:11). Tomando en cuenta estas definiciones, identificamos la modernización agrícola por el uso de insumos industriales como fertilizantes y plaguicidas químicos, maquinaria, equipo de riego y semillas mejoradas. Un rasgo distintivo de esta agricultura, es la energía no renovable que se consume.

${ }^{6}$ Algunos de los impactos que trajo la revolución verde es que al cultivarse grandes extensiones con la misma variedad de semilla, los cultivos se hicieron más vulnerables a las plagas; se emplearon nutrientes en cantidades crecientes para proporcionar a las plantas el alimento que antes obtenían del barbecho o de la alternancia de cultivos. "Los residuos tóxicos en los alimentos, la contaminación en las aguas, la salinización por sobreexplotación de acuíferos; la desprotección de los suelos por la extensión de la actividad agrícola y ganadera; la sobreexplotación de energía fósil y materias primas de los países subdesarrollados para mantener los altos rendimientos de la agricultura de los países ricos; la desaparición de especies y variedades; etcétera, comenzaron a crecer a ritmos superiores a los rendimientos" (González, 1996:430).

7Para Rifkin, la revolución verde ha hecho que aumente la cantidad de gas metano liberado a la atmósfera, lo cuál acentúa el calentamiento global. El gas metano se crea por la composición de sustancias orgánicas que se producen en ambientes deficientes de oxígeno. Esto lo originan sobre todo los arrozales junto con el ganado, que se ha incrementado a partir de la revolución verde (Rifkin, 1990:21).

${ }^{8}$ Esta transformación se apoyó en dos concepciones básicas: "que los procesos productivos agrarios podían ser manipulados mediante la aplicación de conocimientos físico-químicos, y que la sustitución progresiva de trabajo por capital -a semejanza de los procesos industriales-, constituía la manera más adecuada de incrementar la productividad del trabajo; la intensificación productiva, el aumento de insumos externos, el aumento de la escala de explotación, la especialización y la mecanización lo harían posible. En otras palabras, el crecimiento agrario fue considerado como una función del desarrollo tecnológico" (González, 1996:403-404).

${ }^{9}$ El modelo de industrialización basado en la sustitución de importaciones llegó a su fin con la crisis de 1982. México enfrentó esta crisis mediante una política de cambio estructural y transformación productiva, sustentada fundamentalmente en la liberalización de la economía, y el Estado facilitó un libre juego a los mercados en la asignación de recursos. La flexibilización de las reglas de contratación trajo consigo despidos, falta de empleo, reducción de salarios, etc. Por último, el Estado promovió una política de apertura externa, que permitió la subcontratación internacional (Canales, 2002).

${ }^{10}$ Deterioro en la fertilidad de las tierras agrícolas por el uso indiscriminado de agroquímicos. Contaminación de los mantos acuíferos y del agua de riego. Se producen cultivos agrícolas tóxicos por el uso de pesticidas.

${ }^{11}$ Por ejemplo, para los productores con grandes extensiones de tierra, tecnología (maquinaria agrícola y riego) y capital, que acapararon los recursos ofrecidos por la revolución verde, como los caciques locales, empresarios agrícolas y las agroindustrias.

${ }^{12}$ No queremos decir que las pequeñas extensiones de terreno sean una limitante para los productores que busquen ser competitivos y vivir dignamente con lo que produzcan, pero son a los que menos se apoya. A quienes se apoya y cuentan con las ventajas del mercado, es a los grandes latifundistas y empresarios agrícolas. Además, una parcela pequeña no es compatible en muchos de los casos con el estilo de producir moderno, por lo tanto, los productores minifundistas viven dependiendo de lo que dicten los mercados regionales e internacionales, y más cuando no están organizados.

${ }^{13}$ Esto lo podemos constatar en productores pertenecientes a diferentes ejidos del modulo de riego No. 4 de la región del valle de Zamora, en el estado de Michoacán (Gil, 2012).

${ }^{14} \mathrm{El}$ ecosistema incluye tanto a los organismos como al medio abiótico, los cuales están interrelacionados, y gracias a ello, hacen posible la vida en el planeta. El ecosistema es un "(...) sistema compuesto de procesos físico-químicos-biológicos que operan como parte de una unidad espacio-temporal (...)", es entonces “(...) una unidad fundamental de organización en ecología, y una unidad estructural de la biosfera" (Maass, 1990:12). 
${ }^{15}$ Un nuevo paradigma que intenta enfrentar estas deficiencias es la agroecología, la cual, según González, “(...) reivindica la necesaria unidad entre las distintas ciencias naturales entre sí y con las ciencias sociales para comprender la interconexión entre procesos ecológicos, económicos y sociales, reivindica, en fin, la vinculación esencial que existe entre el suelo, la planta, el animal y el hombre" (González, 1996:410).

${ }^{16}$ Para Carabias \& Provencio (1994), el concepto de desarrollo sustentable ha sido utilizado abusivamente, al adaptarlo de una manera oportunista según los intereses de quienes se lo apropian. No obstante, el contenido de su formulación no se ha vaciado, los principios siguen siendo básicos. Asimismo, para Sunkel \& Gligo (1980), el desarrollo sustentable y equitativo debe ser una estrategia a nivel local, regional y nacional que permita la posibilidad a las generaciones venideras de mejorar su calidad de vida sin destruir los recursos naturales del planeta. Debe tener una creciente eficiencia tanto económica como ambiental, tanto en el uso de los recursos naturales como en la producción de bienes y servicios. La tecnología a emplearse debe proteger los recursos naturales utilizando métodos de producción que utilicen insumos menos contaminantes. El desarrollo sustentable debe ser equitativo, el desempleo y la miseria no deben tener cabida en un desarrollo sustentable y equitativo.

${ }^{17}$ Según Torres (1995), algunas causas que propiciaron la crisis del modelo de producción agrícola fueron entre otras: que se diluyó el reparto de tierras, se reorientó el gasto público para favorecer a la industria, la apertura de nuevas tierras perdió impulso, sin que el aumento de nuevas tierras lo compensara; se estancaron los precios de garantía de los principales productos del mercado interno; se disminuyó la demanda de productos de exportación, entre otras causas.

${ }^{18}$ Para González (1996), la agricultura es un ecosistema artificial debido a que el ecosistema natural es total o parcialmente reemplazado “(...) por un conjunto de especies vegetales o animales en proceso de domesticación (...), tales ecosistemas carecen de capacidad de autorreproducirse y necesitan el aporte de energía externa, ya sea humana, animal o fósil. Cada agroecosistema es producto, pues, de una determinada forma de explotación en la medida en que combina de manera específica el trabajo humano, los saberes, los recursos naturales y los medios de producción con el fin de producir (transformando, pero también consumiendo recursos), distribuir y reproducir los bienes necesarios en cada momento histórico para la vida" (González, 1996:410-413).

${ }^{19} \mathrm{Cabe}$ decir, que existen minifundios productivos e improductivos, eficientes e ineficientes, por lo que no se puede homogenizar la situación de éstos. Véase, Torres, G., El minifundio en una estrategia alternativa de desarrollo, 1995.

${ }^{20} \mathrm{El}$ metabolismo entre naturaleza y sociedad “(...) implica el conjunto de procesos por medio de los cuales los seres humanos organizados en sociedad, independientemente de su situación en el espacio (formación social) y en el tiempo (momento histórico), se apropian, circulan, transforman, consumen y excretan, materiales y/o energías provenientes del mundo natural" (Toledo \& González, en prensa). Esto significa que la forma en que los humanos se organizan en sociedad determinará la forma en que afectan, transforman y se apropian de la naturaleza, lo que a su vez condiciona la manera en que las sociedades se configuran. Esta conceptualización supera la habitual separación entre las ciencias naturales y ciencias sociales en la práctica dominante del quehacer científico. De esta manera, las relaciones entre los humanos y la naturaleza son siempre dobles: individuales o biológicas, y colectivas o sociales (lbíd.).

${ }^{21}$ Los factores que han influido en la formación de estas disciplinas híbridas han sido entre otros: el proceso de globalización, el desarrollo del conocimiento especializado, el despliegue de nuevas tecnologías, y a aparición de la crisis ambiental (Toledo, 1999:59). Lo que significa que lo rural sólo es analizable desde un abordaje interdisciplinario o integral, donde es necesario utilizar de manera integrada los enfoques de las ciencias naturales y sociales. El estudio de lo rural no puede estudiarse desconectado del universo urbano-industrial, mucho menos separado del mundo natural (Ídem: 59-60). 


\section{Referencias bibliográficas}

Altieri, M. (2002). "Una perspectiva agroecológica para una agricultura ambientalmente sana y socialmente justa en la América Latina del siglo XXI". En Leff, E. (coord.). La transición hacia el desarrollo sustentable: Perspectivas de América Latina y el Caribe. México: SEMARNAT/INE/UAM/ONU/PNUMA.

Barkin, D. (1997). "La modernización rural y la calidad de la vida: propuesta para el desarrollo colectivo frente a la ofensiva neo-liberal". En López, G. (coord.). Sociedad y medio ambiente en México. México: El Colegio de Michoacán.

Bifani, P. (1997). Medio Ambiente y Desarrollo. México: Universidad de Guadalajara.

Cabanillas, A. (1992). Técnicas de cultivo en agricultura ecológica. Ministerio de Agricultura, Pesca y Alimentación. España.

Calva, J. (1989). Crisis agrícola y crisis alimentaria. México: Fontamara.

Canales, A. (2002). "Migración y trabajo en la era de la globalización: el caso de la migración". Revista Papeles de población № 33. CIEAP/UAEM. julio/septiembre. México.

Carabias, J. (1990). "Las políticas de producción agropecuaria, la cuestión alimentaria y el medio ambiente". En Leff, E. (coord.). Medio ambiente y desarrollo en México. Centro de Investigaciones interdisciplinarias en humanidades/UNAM CIIH. México: Porrúa.

(2002). "Conservación de los ecosistemas y el desarrollo rural sustentable en América Latina: Condiciones, limitantes y retos". En Leff, E. et al. (coord.). La transición hacia el desarrollo sustentable: Perspectivas de América Latina y el Caribe. México: SEMARNAT/INE/ UAM/ONU/PNUMA.

Carabias, J. \& Provencio, E. (1994). "La política ambiental mexicana antes y después de Río". En Glender, A. \& y Lichtinger, V. (coords.). La diplomacia ambiental: México y la Conferencia de las Naciones Unidas sobre Medio Ambiente y Desarrollo. México: SER-FCE.

Best, G. (1990). "Un desarrollo energético alternativo y la gestión del medio ambiente". En Leff, E. (coord.). Medio ambiente y desarrollo en México. México: Centro de Investigaciones Interdisciplinarias en Humanidades/UNAM CIIH/Porrúa.

Eckart, B. (1996). "Mito y naturaleza en Mesoamérica". Etnoecológica, № 0 -5, agosto.

Escalante, R. (1993). "Desarrollo sustentable y unimodal". En Calva, J.L. (coord.) Alternativas para el campo mexicano. Tomo II: Cap. XIII. Fontamara/Fundación Friedrichs Ebert/UNAM, México.

Gil, J. (2012). La costumbre de cultivary moverse al norte. Circuito migroagrícola en el valle de Ixtlán, Michoacán. México: El Colegio de Michoacán/Universidad de La Ciénega del Estado de Michoacán de Ocampo, Zamora/Sahuayo, México.

Gramont, H. (2009). "La Nueva Ruralidad en América Latina". Ponencia Seminario Nueva Ruralidad, Asociación Mexicana de Estudios Rurales, D.F. México.

González, M. (1996). "Los fundamentos agroecológicos de una Historia Agraria alternativa". En Tortolero, A. (coord.). Tierra, agua y bosques: Historia y medio ambiente en el México central. México: Centro Francés de Estudios Mexicanos y Centroamericanos, Instituto de Investigaciones Dr. José María Luis Mora/Potrerillos Editores/S.A. de C.V./Universidad de Guadalajara.

Hogan, J. (2002). "Movilidad poblacional, sustentabilidad ambiental y vulnerabilidad social: Una perspectiva lati№americana". En Leff, E. (coord.). La transición hacia el desarrollo sustentable: Perspectivas de América Latina yel Caribe. México: SEMARNAT/INE/UAM/ONU/PNUMA.

Lampkin, N. (1998). Agricultura ecológica. Ed. Mundi-Prensa. España.

Leff, E. (1997). "Pensar la complejidad ambiental". En Gaceta ecológica, № 44.

Meadows, D. (1972). Los límites del crecimiento. México: FCE.

Olvera, A. (1997). "Transformaciones económicas, cambios políticos y movimientos sociales en el campo. Los obstáculos a la democracia en el mundo rural". En Alonso, J. \& Ramírez, J.M. (coords). La democracia de los de abajo en México. Guadalajara: UNAM/La Jornada Ediciones/Consejo Electoral del Estado de Jalisco.

Riaño, R. \& Keilbach, N. (2009). "Mujeres y nueva ruralidad: un estudio de caso sobre la desfeminización de la agricultura". Revista Sociedades Rurales. Producción y Medio Ambiente. Vol. 9. № 18. UAM/Xochimilco. México.

Rifkin, J. (1990). Entropía: Hacía el mundo invernadero. Barcelona: Urano.

Rubio, B. (1997). "La crisis agrícola en los noventa y la feminización rural en México". En Alberti, P. \& E. Zapata (eds.), Desarrollo Ruraly Género. Estrategias de sobrevivencia de las mujeres campesinas e indígenas ante la crisis económica. México: Colegio de Postgraduados.

Sunkel, O. (1980). "Introducción: la interacción entre los estilos de desarrollo y el medio ambiente en América Latina". En Sunkel, O. y Giglo, N. (coords.). Estilos de desarrollo y medio ambiente en América Latina. Tomo 1. México: FCE (Trimestre económico № 36).

Toledo, V., Álvarez-Icaza, P., \& Ávila, P. (eds.) (1993). Plan Pátzcuaro 2000: investigación multidisciplinaria para el desarrollo sostenido. México: Fundación Friedrich Ebert.

Toledo, V. \& González de Molina, M. (1994). El metabolismo social: las relaciones entre la sociedad y la naturaleza en Las disciplinas socio-ambientales. México: Edit. Trotta.

Toledo, V. (1996). "Saberes indígenas y modernización en América Latina: historia de una ignominia tropical”. Etnoecológica № 45. México.

Toledo, V., Alarcón P. \& Barón L. (1999). "Estudiar lo rural desde una perspectiva interdisplinaria: una aproximación al caso de México". Estudios Agrarios № 12. México.

Torres, G. (1995). El minifundio en una estrategia alternativa de desarrollo, Universidad Autónoma Chapingo, México.

(2006). Poscivilización: guerra y ruralidad. México: Plaza y Valdés/Universidad Autónoma Chapingo.

Torres, G., \& Calderón, J. (1995). Devaluación, crisis y agro. México: Universidad Autónoma Chapingo.

Vivar, J. (2012). "Feminización y valorización del trabajo. Un análisis desde las prácticas laborales y el uso de las herramientas". Textual. Análisis del Medio Rural Latinoamericano № 60. Chapingo. Julio/ Diciembre. México. 